\title{
"Ningún Iroqués era Mohicano": La influencia del contenido en las tareas de razonamiento lógico
}

\author{
Mikel Asensio, J. Martín Cordero, J. A. García Madruga \\ y JAVIER RECIO

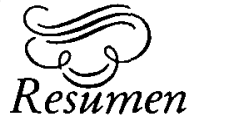

Este estudio se centra en la influencia del contenido de la tarea en problemas de razonamiento deductivo. Se utilizaron cuatro tareas diferentes: de razonamiento proposicional, usando diferentes tipos de conectivas lógicas, silogismos lineales o series de tres términos, silogismos categoriales y la tarea selección de Wason. Para cada una de las tareas se diseñaron dos versiones: una con material temático concreto, diferente para cada tarea y una segunda versión con material abstracto, sin contenido temático concreto. A mbas versiones de la misma tarea eran isomórficas. Se administraron ambas versiones de las cuatro tareas, en el mismo orden comentado, a dos grupos de sujetos: un grupo realizó siempre primero la versión concreta y luego la abstracta y otro grupo realizó siempre la abstracta y después la concreta. Los sujetos fueron alumnos de enseñanza secundaria (3. ${ }^{e r}$ año), 37 y 38 sujetos respectivamente, con una media de edad de 17 años.

Los resultados confirman que no se produce un único efecto facilitador o dificultador del contenido. En una tarea la versión de contenido resulta más fácil, en otra más difíill y en otras dos no hay diferencias. El efecto mayor se produce en aquella tarea en que los sujetos parecen disponer de un "esquema de permiso" muy claro sobre el contenido temático de la tarea. Los resultados se interpretan en base a una hipótesis de doble procesamiento sintáctico y semántico. En cuanto al efecto facilitador del orden de presentación (abstracto-concreto; concreto-abstracto) los resultados indican que la versión concreta facilita la abstracta pero que la abstracta dificulta la ejecución en la concreta. Estos resultados irían en la línea de presuponer una primacía de procesamiento semántico sobre el sintáctico.

\section{Abstract}

This work focuses on the study of the task content influence in deductive reasoning problems. Four different tasks were utilized: propositional reasoning with different types of logical connectives, lineal silogisms or three terms series, categorial silogisms and the Wason selection task. Two versions were designed for each of the proofs: one version with concrete thematic material, different for each proof and another version with abstract material, without any concrete thematic content. Both versions of the same task were isomorpbic. Botb of them were administered for the four tasks, in the same order commented, to two groups of subjects: one group always performed first the concrete version and then the abstract one, and the other group always performed first the abstract and then the concrete one. The subjects were Secondary School pupils ( 3 year), 37 and 38 respectively, being the mean 17 years of age.

The results confirme that there is not a unique facilitative or difficultative effect produced by the content. In a task the content version is easier, in otber more difficult and in the otber two there are no differences. The greatest effect is in that task in which the subjects seem to bave a very clear "permission scheme" about the thematic content of the task. The results were interpreted according to a double, syntactic and semantic, processing bypothesis. As for the facilitative effect of the presentation order (abstract-concrete; concrete-abstract) the results indicate that the concrete version facilitates the abstract one, but that the abstract makes the performance of the concrete one more difficult. These results would go on the line of presupposing a primacy of the semantic processing on the sintactic processing.

Agrale imientos: Esta investigación ha sido posible gracias a una beca concedida por la CAYCYT, dentro del proyecto "Desarrollo cognitivo de la adolescencia a la edad adulta", codirigido por Mario Carretero y Juan Antonio García Madruga, en el que han participado: Mikel Asensio, Jesús Martín Cordero, Amparo Moreno, Puy Pérez Echevarría, Juan Ignacio Pozo y Javier Recio. Queremos agradecer a J. L. González Díaz su colaboración en el tratamiento estadístico de los resultados.

Dirección de los autores: Facultad de Psicología de la Universidad Autónoma de Madrid y Facultad de Psicología de la Universidad Nacional de Educación a Distancia. 


\title{
1. EL RAZONAMIENTO LOGICO Y LA POLEMICA SOBRE LA RACIONALIDAD HUMANA
}

Partamos de un breve ejemplo que nos permita situar el tema que nos proponemos abordar. Imagine el lector la siguiente situación:

\section{Persona 1: «¿Podría indicarme dónde está el Santiago Bernabéu?». \\ Persona 2: "Aquellos de alli son aficionados del Madrid". \\ La persona 1 se dirige al grupo mencionado y les pregunta.}

Tal como señala Johnson-Laird (1983) en un ejemplo similar, la conducta de la persona 1 se ha regido por una cadena de inferencias que podría ser del siguiente tipo:

\author{
"Aquellos son aficionados del Madrid. \\ El Santiago Bernabéu es el estadio del Madrid. \\ Los aficionados del Madrid saben dónde está el estadio de su equipo." \\ Aquellos aficionados del Madrid saben dónde está el estadio Santiago Bernabéu.
}

La primera premisa se deriva de la información aportada por la persona 2, las dos siguientes premisas se derivan del conocimiento del mundo de la persona 1 y la conclusión se deriva de un proceso de razonamiento. Es evidente que procesos como el anterior se producen muy a menudo en la conducta de los seres humanos. Merece la pena resaltar varios aspectos del ejemplo propuesto: a) se trata de un proceso de inferencia que cualquier persona normal reconocería como válido, b) es un proceso que tiene lugar a través del lenguaje, aunque tal vez no sea éste el medio último en el que se realiza la transformación que da lugar a la deducción, c) desde cierto punto de vista puede resultar tentador formular este proceso (el que va desde la última premisa a la conclusión) mediante alguna expresión de fuerte aroma lógicoformal, tal como «Todo A es B. Algún A es Bi».

Los procesos de razonamiento tienen una importancia crucial para dar cuenta de comportamientos tales como la toma de decisiones, la solución de problemas, la elaboración de hipótesis, la valoración de supuestos e informaciones diversas y, desde luego, cumplen un papel esencial en el desarrollo del conocimiento científico y tecnológico. La capacidad humana para inferir conclusiones a partir de unas premisas dadas ha concitado desde la antigüedad la atención de pensadores y estudiosos. Es claro que la dilucidación de los procesos por los que se modifica la información en el razonamiento ha de ocupar un lugar central en cualquier teoría sobre el funcionamiento de la mente humana. Tradicionalmente ha sido la lógica formal el candidato más firme a ocupar la plaza de modelo sobre el que analizar los procesos de razonamiento. Sin embargo, también han existido posiciones que cuestionan la bondad de esta perspectiva y plantean teorías alternativas para dar cuenta del razonamiento lógico. Una visión global del campo del razonamiento, de las tareas más usuales y de los problemas de la racionalidad, la influencia del contenido y la comprensión de la tarea, puede verse en García Madruga y Carretero (1986).

A riesgo de simplificar en exceso la cuestión puede decirse que la situación relativa de los diversos enfoques viene definida por la respuesta que dan a la 
siguiente pregunta: « Es necesario un componente lógico para explicar el razonamiento humano? Caso de ser afirmativa, la respuesta conduce a postular la candidatura de alguna lógica en los procesos de razonamiento. Así, por ejemplo, Piaget, en el último y más avanzado de sus estadios del desarrollo cognitivo, postula que los sujetos alcanzan una competencia lógica que les permite realizar operaciones formales con independencia del contenido de las tareas. Esta concepción piagetiana sobre el importante papel que cumple la lógica proposicional en la descripción de la competencia cognitiva del sujeto en el período formal, aunque reducida en su alcance y matizada en sus últimos escritos (Piaget, 1970), puede ser ilustrada con una conocida cita sobre los procesos mentales que tienen lugar cuando el sujeto se enfrenta a una situación compleja (en una tarea de razonamiento).

«El sujeto se hace a sí mismo dos tipo de preguntas: a) si el hecho ' $x$ ' implica el hecho ' $y$ '... Para verificarlo considerará en este caso si existe o no un contraejemplo de ' $x$ ' $y$ 'no-y'; b) también se preguntará si, por el contrario, es ' $y$ ' lo que implica ' $x$ '...'”

(Piaget, en Beth y Piaget, 1961, p. 18, de la versión castellana)

Sin que esta cita suponga necesariamente que el sujeto, para Piaget, cuenta con un completo arsenal de lógica proposicional, sí parece claro que según este autor el problema se elabora haciendo uso de algún tipo de esquema formal de inferencia. Más adelante abordaremos algunas propuestas récientes de lo que ha dado en llamarse «lógica mental».

Volviendo a la pregunta que planteábamos, también es posible contestarla negativamente, y postular que no es necesario suponer un componente lógico en el mecanismo de razonamiento humano. Como veremos, es posible plantear la existencia de estructuras ligadas al conocimiento, esquemas, que den cuenta del comportamiento inferencial de los sujetos. Asimismo, existe la alternativa de postular un proceso semántico sensible a los contenidos, pero menos ligado a éstos que los esquemas; nos referimos a los modelos mentales.

Una de las características generales que tienen en común los puntos de vista partidarios de la lógica mental es la necesidad de explicar los frecuentes errores que es posible detectar en el rendimiento de los sujetos en tareas de razonamiento lógico. La versión más tradicional de esta explicación es la elaborada por Henle $(1962,1978)$. Según esta teoría, los errores que se observan en el razonamiento no son, en realidad, producto de la lógica subyacente al proceso de razonamiento en sí, sino más bien son provocados por el hecho de que los sujetos olvidan o reinterpretan idiosincrásicamente la información contenida en las premisas, o porque añaden suposiciones injustificadas durante el razonamiento. En definitiva, una comprensión defectuosa podría explicar buena parte de los errores en el rendimiento de los sujetos. Las personas son capaces de razonar de acuerdo con la lógica, dirían los partidarios de este punto de vista, y si en ocasiones cometen errores, no se debe a la competencia lógica, sino a otros procesos. Tal como señala Johnson-Laird (1983), la fuerza de este tipo de argumento, que ha ejercido una gran influencia sobre los psicólogos que investigan el razonamiento, reside en el hecho de que descansa en la convicción inconmovible de la racionalidad del pensamiento consciente. Es posible que una de las ventajas de este argumento sea el haber llamado la atención sobre la importancia que tienen los procesos de comprensión en el 
razonamiento, pero parece indudable que da lugar a una posición inatacable, dado que se parte de que la mejor manera de refutar la explicación de un hecho es negar el propio hecho: los errores del razonamiento, cuando se producen, no son achacables al razonamiento en sí, sino a algún otro proceso.

A partir de las reflexiones anteriores podría pensarse que el campo queda distribuido entre partidarios de la lógica mental y, consecuentemente, defensores de la racionalidad, por un lado, y negadores de la lógica mental que al tiempo son detractores de la racionalidad humana. Tal visión sería errónea. Sería más justo decir que existen partidarios de la existencia de estructuras formales, cuya entidad queda a salvo de los errores observados en el rendimiento, dado que se supone que éstos tienen lugar en otros procesos distintos del razonamiento y, frente a ellos, se sitúan quienes explican los procesos inferenciales mediante algún tipo de estructura más o menos cercana a los contenidos de la tarea, pero sensible a éstos. Negar la existencia de una lógica mental, entendida como procedimiento sintáctico formal, no significa negar la posibilidad de racionalidad a los humanos, sino matizarla incluyendo en una misma teoría la realización de inferencias válidas, la comisión de errores y la existencia de sesgos en el razonamiento.

A pesar de que recientemente se ha presenciado el florecimiento de posiciones más cercanas a los contenidos y a la semántica para explicar los procesos de razonamiento, no puede decirse que los partidarios de algún tipo de estructura sintáctica, ajena a los contenidos, sean poco numerosos. En efecto, en los últimos años han aparecido en la literatura diversas propuestas en este sentido (Braine, 1978; Braine y Rumain, 1983; Cohen, 1981; O’Brien y Overton, 1980, 1982; Overton et al., 1987; Byrnes y Overton, 1988; Osherson, 1975; Rips y Marcus, 1977; Rips, 1983; entre otros). Posiblemente, sea la posición de Braine y sus colaboradores la más completa e interesante de las mencionadas. Seguidamente expondremos los aspectos más relevantes de la teoría de los esquemas de inferencia propuesta por Braine.

Comenzaremos por exponer brevemente el tipo y la amplitud de la competencia lógica que Braine propone para el razonamiento humano, para seguidamente contemplar cómo aborda el problema de los errores en el razonamiento. Según Braine, el razonamiento actual procede mediante reglas de inferencia, éstas serían "reglas que indican que una cierta proposición puede concluirse de modo inmediato cuando ciertas otras proposiciones han sido establecidas» (Braine, 1978, p. 3); por lo tanto, lo esencial es conocer la forma de estas reglas de inferencia: los esquemas de reglas de inferencia son fórmulas que definen reglas de inferencia mediante la especificación de su forma (op. cit. p. 3). Es decir, las reglas de inferencia serían instanciaciones de los esquemas de inferencia. Para Braine y Rumain (1983), es posible definir hasta 17 de estos esquemas de inferencia en el razonamiento proposicional «natural». Esta competencia lógica supone todo un repertorio de inferencias posibles, entre las que se incluyen el Modus Ponens, diversos esquemas de razonamiento sobre alternativas $y$, en general, todos aquellos de los esquemas descritos por Braine que implican un razonamiento directo; es decir, la posibilidad de extraer directamente una conclusión de un conjunto de premisas. Los esquemas de razonamiento se adquieren como parte del aprendizaje de la lengua. La propuesta de Braine es completa y compleja y no es ésta la ocasión de realizar una exposición detallada de la misma; baste con resaltar que la aplicación de estos esquemas de inferencia, que suponen la competencia lógica 
íntima, resulta indiferente a los contenidos a los que se aplica (dado que los esquemas son formales y constituyen una sintaxis) y está mediada por la utilización de ciertas unidades linguísticas tales como: «y», «0», «si...», «todos», «cada», «algunos", etc.

Tal como indicábamos más arriba, la afirmación de que los errores del razonamiento no tienen su origen en el propio proceso de inferencias, sino en un proceso previo de comprensión de la tarea, es el recurso tradicional para abordar este problema desde las tesis partidarias de la lógica mental. En este caso, Braine y Rumain (1983) parten de la distinción trazada por Donaldson (1976) entre dos tipos de comprensión del lenguaje. Por un lado estaría la "comprensión ordinaria», a la que se aplicarían plenamente las convenciones generales descritas por Grice $(1975,1978)$. Este tipo de comprensión ordinaria o cotidiana supone que la motivación esencial del oyente es descifrar lo que el hablante desea comunicar, es un proceso rápido y automático en el que el oyente utiliza profusamente su conocimiento del mundo y realiza frecuentes inferencias que permiten completar los aspectos no explícitos del mensaje recibido. Quizás la convención más relevante que entra en juego respecto al problema que nos ocupa sea el "principio cooperativo», según el cual el oyente supone que el hablante procura ser informativo, relevante y verdadero en sus comunicaciones. En este sentido Braine y Rumain (1983) entienden que muchas de las tareas típicas de razonamiento lógico violan a menudo el principio cooperativo.

El segundo tipo de comprensión o "comprensión analítica» es un proceso diferente, mediante el cual se intenta descifrar el contenido del discurso «en sí». Este parece ser un proceso relativamente especializado que se desarrolla en campos tales como el derecho, la escritura de tipo científico y otros. En todo caso, lo que interesa de la distinción es que la comprensión analítica para Braine y Rumain supone deshacerse de las convenciones usuales mencionadas anteriormente y centrarse en las implicaciones y consecuencias del mensaje literal, realizando un proceso más lento y cuidadoso que el que tiene lugar en la comprensión ordinaria. De las características de ambos tipos es fácil desprender que si, tal como se ha planteado, muchas de las tareas de razonamiento lógico violan el principio cooperativo y exigen una comprensión de tipo analítico para poder ser realizadas con éxito, contamos con una nueva versión de la teoría de la comprensión como causa de los errores en el razonamiento. En efecto, desde la perspectiva de Braine y Rumain, los sujetos utilizan habitualmente la comprensión ordinaria para enfrentarse a tareas de razonamiento de inocente apariencia. Sin embargo, creemos que esta explicación tiene problemas. Si la «verdadera» competencia lógica de las personas únicamente sale a relucir cuando éstas se entregan al lento y especializado proceso de la comprensión analíica, en tanto que la comprensión ordinaria se encuentra viciada (desde el punto de vista de la competencia lógica) por sesgos, inferencias que pueden no estar justificadas, e irracionalidades de toda catadura, ¿no sería más correcto plantear que la «verdadera» competencia lógica es cosa de especialistas?, ¿no resultaría más parsimoniosos aceptar que el razonamiento lógico es una habilidad que depende del dominio al que se aplica y que puede ser desarrollada?

Anteriormente indicábamos que también han sido formuladas alternativas a la posición de la lógica mental. Una de las perspectivas que postulan estructuras de razonamiento sensibles a los contenidos es la de los «esquemas 
pragmáticos de razonamiento». Este punto de vista ha sido defendido por autores como Cheng y Holyoak (1985), Fong, Krantz y Nisbett (1986), Holland, Holyoak, Nisbett y Thagard (1987), etc. Los esquemas pragmáticos de razonamiento son estructuras de conocimiento inducidas a partir de las experiencias de la vida diaria. Son un conjunto de reglas generalizadas sensibles al contexto que, al contrario que las reglas puramente sintácticas, se definen en términos de clases de objetivos (tales como llevar a cabo acciones deseables y realizar predicciones sobre sucesos futuros) y relaciones entre estos objetivos (tales como causa y efecto, requisito y acción permitida). Por lo tanto, los esquemas pragmáticos de razonamiento sin estar vinculados a un contenido específico, sí lo están a un rango de contenidos, de este modo han sido definidos esquemas tales como los de "permiso", "obligación», "relación causal». Las reglas vinculadas a estos esquemas no son reglas sintácticas abstractas, sino reglas generales o heurísticos destinados a resolver tipos generales de problemas. Así, por ejemplo, un esquema de «permiso» indica al sujeto que lo utiliza para enfrentarse a una tarea condicional, cuya estructura lo ha evocado, que para realizar el comportamiento descrito en el condicional habrá de satisfacerse el requisito que éste implica y, a la inversa, si no se ha satisfecho el requisito, no es posible realizar el comportamiento.

En efecto, supongamos que proponemos una regla condicional del siguiente tipo: «Si una persona ocupa un alto cargo, entonces está cercana al partido del gobierno». Esta regla indica que el requisito para ocupar un alto cargo es encontrarse cercano al partido del gobierno, y puede decirse que «sesga» hacia el modus tollens, dado que cualquier persona sensata concluiría a partir de la misma que si alguien no está cercano al partido del gobierno, difícilmente ocupará un alto cargo; por otra parte, interpretar la regla a partir del esquema pragmático de permiso también bloquea la famosa falacia de afirmación del consecuente, ya que muchos son los cercanos al partido del gobierno y, como es bien sabido, no todos ellos alcanzan un alto cargo. Por último, el esquema también bloquea la falacia de negación del antecedente, dado que respecto a lo afirmado en el ejemplo, poco importa si aquellos que no ocupan un alto cargo se hallan o no cercanos al partido gobernante. Este ejemplo ilustra cómo la utilización de un esquema pragmático de permiso conduce, en este caso, a razonar de un modo acorde con la lógica de proposiciones y a realizar conclusiones razonables sobre la posibilidad de acceder a un alto cargo y, todo ello, sin hacer uso de esquemas de inferencia formales.

En nuestra opinión, los esquemas pragmáticos de razonamiento permiten abordar con mayor facilidad que la perspectiva sintáctica fenómenos tales como los errores que a menudo se detectan en tareas de razonamiento lógico y el efecto facilitador que ocasionalmente se observa al emplear contenidos temáticos en algunas tareas como el condicional. Tanto los errores como el efecto facilitador de ciertos contenidos vendrían determinados por la posibilidad de «encajar" el material propuesto por la tarea de razonamiento en algún esquema pragmático. Sin embargo, este enfoque sigue planteando problemas para explicar por qué algunos sujetos sí son capaces de resolver adecuadamente tareas complejas de razonamiento lógico con materiales abstractos. Una posibilidad sería que el reducido porcentaje de individuos capaces de resolver adecuadamente ciertos silogismos, por ejemplo, hubieran actuado a partir de la asimilación previa de las reglas formales de razonamiento silogístico, pero esto parece poco plausible. Otra dificultad en relación con los esquemas 
pragmáticos de razonamiento es que no parece muy factible abordar mediante ellos procesos tales como el razonamiento con silogismos y otras tareas distintas del condicional.

Hasta ahora hemos considerado dos propuestas alternativas a la explicación del razonamiento humano. Por un lado existe la posibilidad de suponer que un componente lógico de algún tipo actúa en la cabeza de nuestros sujetos cuando éstos razonan; un ejemplo de este punto de vista son los esquemas de reglas de inferencia defendidas por Braine y sus colaboradores. Hemos visto que estas posiciones han de hacer frente a la existencia de errores en el razonamiento y a los efectos diferenciales que sobre él tienen los distintos contenidos. Por otra parte, también hemos examinado la posibilidad de explicar el rendimiento de los humanos en tareas de razonamiento desde estructuras no sintácticas, más sensibles al contexto. Por ejemplo, los esquemas pragmáticos de razonamiento propuestos por Cheng, Holyoak y otros. Parece que, aun constituyendo una explicación más amplia de los efectos observados, no es posible dar cuenta desde ellos en la competencia lógica de las personas. No constituyen un modelo de la competencia humana. Seguidamente y para finalizar este apartado, consideraremos una tercera posibilidad que nos parece más prometedora: los modèlos mentales.

Lo que necesitamos es un mecanismo de razonamiento que sea sensible a los contenidos y que, por tanto, pueda dar cuenta de los efectos temáticos, pero que al tiempo no esté íntimamente vinculado a ellos, que sea lo suficientemente general como para poder explicar cómo es posible que las personas sean capaces de razonar válidamente sobre contenidos nuevos, en los que no han tenido experiencia previa. Un mecanismo que permita integrar en una misma teoría los procesos de razonamiento válido y los errores y sesgos que a menudo se observan. El contexto teórico de los modelos mentales propuestos por Johnson-Laird $(1983,1988)$ es de una extremada complejidad, y en él intervienen conceptos, hipótesis y teorías de orígenes tan diversos como la psicología experimental, la filosofía, la semántica y la inteligencia artificial.

El aspecto esencial para el razonamiento de la teoría de los modelos mentales es que un sistema de inferencia puede comportarse de un modo completamente lógico a pesar de no utilizar reglas de inferencia, esquemas de inferencia o cualquier otro mecanismo correspondiente a un cálculo lógico" (Johnson-Laird, 1983, p. 131). Esta perspectiva indica que el razonamiento consiste en la construcción de modelos mentales a partir de las premisas y en la búsqueda de modelos alternativos que podrían hacer que las conclusiones iniciales resultaran falsas. En otras palabras, se trata de una teoría semántica del razonamiento. En efecto, al decir que es una teoría semántica estamos llamando la atención sobre el hecho de que las personas razonan mediante la comprensión de la información incluida en las premisas, esta información es representada construyendo un modelo mental del estado de cosas descrito en las premisas, para seguidamente extraer una conclusión informativa; es decir, que no se encuentre explícita en las premisas. Esta conclusión es puesta a prueba mediante la búsqueda de contraejemplos, es decir, buscando otros modelos mentales en los que las premisas continúen siendo verdad pero la conclusión sea falsa. Se trata, insistimos, de una teoría semántica del razonamiento, dado que el conjunto del proceso se encuentra guiado semánticamente por procesos de comprensión en los que lo que se representa es la información contenida en las premisas y no la forma de ésta. 
Los modelos mentales incluyen una representación de las relaciones funcionales que se dan en la "realidad», es por esto que hablamos de «modelos», porque son representaciones funcionales y discretas. Los modelos se construyen tanto a partir de la información que ingresa en el sistema, como a partir de la información que el sistema extrae de la memoria a largo plazo y que utiliza para el proceso de comprensión, de manera que el modelo de un objeto específico de la realidad será tanto más complejo cuanto más amplio sea el conocimiento previo que sobre él tenga el individuo. De aquí que los efectos de los contenidos temáticos puedan explicarse con relativa facilidad a partir de la teoría de los modelos mentales. En cuanto a los errores en el razonamiento lógico, tendrían dos fuentes principales: o bien las operaciones requeridas en el proceso de deducción implican la construcción de varios modelos mentales y superan la capacidad de la memoria de trabajo (como ocurre con los silogismos más difíciles, que suponen la construcción de tres modelos), o bien el(los) modelo(s) elaborado(s) no es(son) lo suficientemente complejo(s) para dar cuenta de las relaciones funcionales (lógicas) que serían necesarias para alcanzar el nivel de criterio en la tarea propuesta. Otra explicación posible para la comisión de errores en el razonamiento sería que los sujetos a menudo fracasan en la búsqueda de contraejemplos, aunque matizando desde un punto de vista teórico, ésta podría ser una derivación de la insuficiente complejidad del modelo construido por el sujeto.

Las tres posiciones descritas hasta ahora (lógica mental, esquemas pragmáticos y modelos mentales) constituyen el contexto teórico de la investigación que presentamos. Desde un punto de vista computacional podría decirse que el enfoque de la lógica mental incorpora un soporte lógico (un programa) bastante amplio al mecanismo que se encarga del razonamiento; los esquemas pragmáticos constituyen una posición más cercana a lo que se conoce como sistemas expertos, e incluyen una jerarquía adquirida de conocimientos más o menos específicos y, finalmente, los modelos mentales suponen una serie de procedimientos de propósito general que pueden concebirse como los requisitos mínimos de un mecanismo que haya de realizar inferencias. Es posible que en algún momento todas las personas razonen de acuerdo con cada una de las posiciones descritas y que éstas no sean tan excluyentes como a primera vista podría pensarse. Sin embargo, como teoría general parece que los modelos mentales hubieran de enfrentarse a menos dificultades para incorporar la diversidad y complejidad de los datos que las investigaciones sobre razonamiento lógico han generado.

\section{EFECTOS DEL CONTENIDO EN ALGUNAS TAREAS DE RAZONAMIENTO LOGICO}

En el apartado anterior hemos hecho referencia en varias ocasiones al "efecto temático», «los efectos facilitadores de los contenidos» y «los efectos diferenciales de los contenidos de la tarea sobre el razonamiento". Con estas expresiones hacíamos referencia a un conjunto de fenómenos que han sido observados desde hace bastantes años y que pueden resumirse mediante la pregunta: ¿ Ejercen los contenidos de las tareas algún efecto sobre los procesos y resultados del razonamiento? La respuesta a esta pregunta ha de ser afirmativa. Sin embargo, el sentido de ese efecto (facilitación/dificultación) y las caracte- 
rísticas de la influencia realizada sobre los procesos de inferencia, constituyen un asunto mucho más complejo.

Una de las tareas de razonamiento sobre la que más se ha investigado el efecto de los contenidos es la llamada «tarea de selección» de Wason o «tarea de las cuatro tarjetas». Dado que en un apartado subsiguiente describimos la naturaleza de esta tarea, nos contentaremos ahora con indicar que se trata de un problema de razonamiento condicional en el que se emplea una regla de forma " $\mathrm{p} \rightarrow \mathrm{q}$ ". Una de las circunstancias que más llamó la atención de los investigadores sobre esta tarea es que, cuando se utilizan materiales abstractos en ella (por ej., letras y números), el rendimiento de los sujetos no suele superar el $10 \%$ de respuestas correctas. Este dato es llamativo porque las distintas posibilidades de los silogismos condicionales constituyen operaciones que las personas emplean a menudo y con bastante eficacia. En el proceso de indagar este interesante resultado se pensó que tal vez la utilización de contenidos temáticos, más cercanos a la experiencia de los sujetos, produjera una mejora en el rendimiento de éstos. Así ocurrió, a comienzo de los años 70 se informó de diversas investigaciones (Wason y Shapiro, 1971; JohnsonLaird y otros, 1972; Bracewell y Hidi, 1974; Van Duyne, 1974; etc.) en las que había sido posible incrementar muy notoriamente la proporción de respuestas correctas haciendo uso de materiales cuyos contenidos expresaban reglas más o menos familiares para los individuos investigados. Inicialmente se pensó que este efecto facilitador tenía lugar porque los contenidos empleados, al resultar familiares a los sujetos, facilitaban para éstos la búsqueda de contraejemplos que constituye el aspecto más complejo de la tarea de selección. No obstante, como señala Griggs (1983) en su excelente revisión sobre este tema, poco más tarde se realizaron investigaciones en las que el efecto facilitador de los contenidos temáticos parecía haberse perdido o, al menos, haber perdido intensidad (Mantktelow y Evans, 1979; Pollard, 1981; Griggs y Cox, 1982; etc.).

Como se verá más adelante, nosotros mismos hemos obtenido resultados claros en favor de la facilitación en nuestra versión de contenido temático. Sin embargo, lo que resulta evidente a partir de investigaciones realizadas es que la utilización de contenidos temáticos no siempre facilita la realización de la tarea. Cuando los contenidos temáticos dan lugar a una facilitación podemos pensar que ésta puede deberse a dos razones: a) el sujeto puede haber tenido experiencia con reglas específicas iguales o muy similares a la que se empleó en la tarea de razonamiento. En este caso, puede haber ocurrido que la experiencia del sujeto con la regla empleada le haya indicado directamente la pertinencia de la selección del contraejemplo, b) el material empleado puede haber evocado en el sujeto la utilización de una estructura de conocimiento cuyo «comportamiento lógico" resulte adecuado para los requisitos de la tarea. Es lo que Cheng y Holyoak llaman un esquema pragmático, o lo que JohnsonLaird denominaría como modelo mental. Lo importante es que, sea por la experiencia específica con la regla, sea por un esquema o modelo, en ambas circunstancias pueden generarse patrones de respuesta que resultan acordes con la tabla de verdad del condicional como conectiva lógica. Ello no es sorprendente, dado que, incluso un conocimiento tan abstracto como la lógica formal, ocasionalmente encuentra criterios de validez/untilidad análogos en el razonamiento humano.

Sin embargo, tal como adelantábamos, la utilización de contenidos temá- 
ticos en las tareas de razonamiento también ha dado lugar a resultados en los que no era posible apreciar un efecto facilitador, o incluso podía observarse un descenso en el rendimiento de los sujetos. El primero de los casos tiene lugar cuando los sujetos no han tenido experiencia previa alguna con el material que se emplea en la tarea, o cuando éste no permite construir una representación comprensiva del mismo, bien sea por sobrecarga de la memoria de trabajo, bien porque resulta imposible trazar la analogía necesaria que lo vincule a alguna estructura de conocimiento significativa que pueda emplearse en su resolución. En cuanto a aquellos resultados que indican que el empleo de materiales temáticos puede incluso dificultar más la tarea que el uso de materiales abstractos, la mayoría de los datos tienen su origen en el razonamiento silogístico. En efecto, durante la segunda guerra mundial y en los años siguientes se realizaron investigaciones que empleban tareas de razonamiento silogístico en el contexto teórico, muy en boga en aquellos tiempos, de la influencia que las actitudes pueden ejercer sobre el comportamiento. Autores como Janis y Frick (1943), Morgan y Morton (1944) y Lefford (1946) realizaron trabajos en los que se obtuvieron datos que sugerían un fuerte sesgo de los sujetos hacia aquellas conclusiones que se encontraban más acordes con sus creencias personales cuando los contenidos de la tarea ofrecían temas polémicos, sobre los que los sujetos disponían de opiniones previas. Alternativamente, se apreció que los sujetos tendían a seleccionar con menor frecuencia aquellas conclusiones válidas que fueran contrarias a sus creencias. Si bien algunos de estos resultados han sido matizados y criticados metodológicamente (por ej., Henle y Michael, 1956), parece claro que las actitudes de las personas sobre cuestiones polémicas sobre las que se posean opiniones arraigadas pueden ejercer una influencia negativa (desde la perspectiva de la validez) sobre sus procesos de razonamiento.

A partir de las investigaciones que hemos comentado parece claro que, desde luego, los contenidos de las tareas de razonamiento pueden ejercer una influencia importante sobre los procesos de inferencia de los sujetos. La cualidad e intensidad de esa influencia es un problema teórico que pensamos aún tardará en resolverse, si es que esto llega a ocurrir algún día; entretanto hemos procurado avanzar en la dilucidación de estos procesos estudiando el efecto de incluir contenidos temáticos en diversas tareas de razonamiento, de ello trata la investigación que seguidamente presentamos.

\section{DISEÑO EXPERIMENTAL}

Para investigar la influencia del contenido sobre las tareas de razonamiento nos interesaba seleccionar un conjunto de tareas que fuesen suficientemente conocidas de modo que existiera una descripción más controlada de las ejecuciones de los sujetos y, por tanto, se facilitara el análisis de los errores. Se seleccionaron cuatro tipos de tareas consideradas como clásicas en los estudios de razonamiento y sobre las que existe un considerable volumen de trabajo experimental. Fueron las siguientes: la utilización de las conectivas lógicas o lógica proposicional (tarea 1); las series de tres términos o silogismos lineales (tarea 2); los silógismos categóricos (tarea 3); y la tarea de selección de Wason conocida como la prueba de «las cuatro tarjetas» (tarea 4). Todas ellas eran tareas que habían sido estudiadas por nosotros mismos con anterioridad. 
Para cada uno de los cuatro tipos de tareas se diseñaron dos pruebas: la forma A consistía en una versión de contenido tradicionalmente considerado como abstracto (números, letras y colores) utilizando situaciones descontextuadas; la forma $C$ consistía en una versión de contenido concreto, es decir, una versión con contenido, donde la tarea estaba contextuada en situaciones de la vida cotidiana y los ítems recogían relaciones potencialmente significativas entre los elementos. Ambas formas A y C presentaban una estructura lógicoformal isomórfica, de modo que la diferencia en las ejecuciones de los sujetos pudiera deberse exclusivamente a la presencia o ausencia de contenido significativo.

Se utilizó un diseño simple de grupos aleatorios. Ambos grupos solucionaron todas las tareas. El orden de presentación de las tareas fue el mismo para ambos grupos: conectivas, series, silogismos y selección. El orden de presentación de la variable independiente «abstrato-contenido» estaba contrabalanceado, es decir, se variaba de un grupo a otro el orden de presentación de las dos formas de cada tipo de tarea de modo que el grupo 1 siempre realizaba la forma abstracta antes que la concreta (Tabla I).

\section{TABLA I}

Orden de presentación de las dos formas de los cuatro tipos de pruebas para cada uno de los grupos

\begin{tabular}{lcccc}
\hline & Conectivas & Series & Silogismos & Selección \\
\hline Grupo 1 & $1 \mathrm{C}-1 \mathrm{~A}$ & $2 \mathrm{C}-2 \mathrm{~A}$ & $3 \mathrm{C}-3 \mathrm{~A}$ & $4 \mathrm{C}-4 \mathrm{~A}$ \\
Grupo 2 & $1 \mathrm{~A}-1 \mathrm{C}$ & $2 \mathrm{~A}-2 \mathrm{C}$ & $3 \mathrm{~A}-3 \mathrm{C}$ & $4 \mathrm{~A}-4 \mathrm{C}$ \\
\hline
\end{tabular}

Este diseño permitía dos tipos de comparaciones. De una parte podríamos comparar la influencia del contenido comparando las ejecuciones de los sujetos en primera presentación de cada tipo de tarea para cada uno de los grupos (comparación vertical en la Tabla I). Y de otra parte podríamos comprobar la influencia de la ejecución de la versión abstracta sobre la de contenido y viceversa, comparando las presentaciones de las dos formas para cada uno de los grupos (comparaciones horizontales de la Tabla I).

\subsection{Hipótesis}

En general las ejecuciones de las tareas por los sujetos deberían acomodarse a los resultados encontrados en anteriores investigaciones sobre las mismas tareas. Tanto los aciertos como los errores deberían ser los mismos que los descritos anteriormente.

De acuerdo con la discusión presentada en los puntos 1 y 2 , presuponíamos que la inclusión de un contenido significativo en el razonamiento supone la posibilidad de activación de un esquema o modelo (de contenido o declarativo) que facilita la ejecución de la tarea. De esta forma, preveíamos que, de ser cierta la propuesta de la existencia de esquemas o modelos pragmáticos y semánticos en el razonamiento, la inclusión de contenidos en las tareas de 
razonamiento lógico favorecería su solución. Así, las comparaciones verticales deberían ofrecer que la ejecución de las tareas del grupo 1 debería ser peor que la del grupo 2 para la primera forma en los cuatro tipos de tareas presentados.

Respecto a las comparaciones horizontales nos encontramos ante la siguiente disyuntiva. De ser cierta la presuposición de la existencia de un componente pragmático-semántico del razonamiento, se verían favorecidos aquellos sujetos que han realizado previamente la tarea con contenido, es decir, los sujetos del grupo 1. Mientras que de ser cierta la presuposición de un componente sintáctico del razonamiento, se verían favorecidos aquellos sujetos que han realizado previamente la tarea de tipo abstracto, es decir el grupo 2. Evidentemente, este razonamiento parte de un axioma previo, presuponer que la activación de una tarea anterior facilita la ejecución de tareas isomórficas sucesivas.

\subsection{Sujetos}

- El número total de sujetos fue de 75 , distribuidos por igual respecto al sexo. Todos ellos cursaban $3 .^{\circ}$ de BUP, con una edad media de 17 años. Las condiciones socioeconómicas y pedagógicas pueden considerarse como de clase media-alta. Los sujetos estaban naturalmente divididos en dos grupos: el grupo 1 estaba formado por 37 sujetos y el grupo 2 por 38 .

\subsection{Pruebas}

\section{Prueba 1: Conectivas}

La prueba consiste en seis ítems que recogen la utilización de las siguientes conectivas lógicas: conjunción, disyunción, doble negación, incompatibilidad, bicondicional y condicional.

La prueba consta de un cuadernillo de instrucciones y una hoja de respuestas. En el cuadernillo se informa al sujeto de una situación en la que hay una serie de elementos que hay que agrupar de acuerdo con unas reglas establecidas. Estas reglas hacen referencia a cada una de las distintas conectivas. En la hoja de respuestas, cada ítem está formado por una regla y cuatro agrupamientos posibles. El sujeto debe decidir cuáles cumplen la regla y cuáles no, es decir, cuáles acepta y cuáles rechaza.

Forma abstracta. Prueba 1A: La situación creada para esta forma hace referencia a unas fichas de distintos colores que deben agruparse de acuerdo con cada una de las reglas formando montones. Se ofrecen cuatro montones distintos para cada regla y el sujeto debe seleccionar los que la cumplen y los que no la cumplen. Por ejemplo, una regla podría ser «que los montones contengan fichas rojas y amarillas» y los montones propuestos «amarillas y azules», «rojas y amarillas», «azules y rojas» y «amarillas y azules». El alumno debe decidir cuáles de estos montones cumplen la regla y cuáles no la cumplen.

Forma con contenido. Prueba 1C: En este caso se pide al sujeto que haga de jurado en la admisión de grupos para un concurso musical. Estos grupos deben estar formados por una serie de instrumentos de acuerdo con cada una 
de las reglas. Por ejemplo, una regla podría ser «que los grupos tengan batería o piano" y los grupos propuestos «batería y guitarra», «batería y saxo», "piano y guitarra» y «saxo y guitarra». El sujeto debe decidir cuáles de los grupos admite para participar en el concurso y cuáles no.

\section{Prueba 2: Series de tres términos}

La prueba consta de un cuadernillo de instrucciones y una hoja de respuestas. En el cuadernillo se explica el tipo de problemas que se van a plantear y cómo resolverlos, a través de un ejemplo. Se seleccionaron problemas de silogismos lineales del nivel más alto de dificultad.

Formas abstracta. Prueba 2A: En esta forma se presentan los doce problemas en la forma habitual de las series de tres términos, es decir, utilizando las letras A, B y C para representar los términos del silogismo lineal y usando los adjetivos del tipo «mejor que» 0 "peor que» para relacionarlos. Los problemas se resuelven contestando a la pregunta ¿cuál es el mejor? o ¿̇cuál es el peor?, según el caso. Por ejemplo, se muestra al sujeto la siguiente serie «A es mejor que $\mathrm{B}$ » «B es mejor que $\mathrm{C}$ » y se le pregunta: «¿Cuál es el mejor?»

Forma con contenido. Prueba 2C: En el cuadernillo se explica al sujeto que él es el entrenador de unos equipos deportivos y que debe seleccionar a los alumnos de un colegio para formar dichos equipos. Esta selección la hará teniendo en cuenta la información dada en una serie de afirmaciones que ponen en relación a unos alumnos con otros. Estas afirmaciones se harán en forma de series de tres términos en las que los elementos son los alumnos, por tanto, el sujeto deberá contestar en cada problema quién es el alumno mejor/peor, y por tanto a quién seleccionaría para jugar en el equipo. Por ejemplo, se presenta la siguiente serie "Luis es mejor que Pedro" "Pedro es mejor que Antonio» y el sujeto debe contestar a la pregunta "¿cuál es el mejor?».

\section{Prueba 3: Silogismos}

La prueba consta de un cuadernillo y una hoja de respuestas. En el cuadernillo se dan las instrucciones y un ejemplo de cómo se resuelven los problemas. En la hoja de respuestas se plantean diez silogismos de los que el sujeto debe dar la conclusión. Los silogismos escogidos pertenecen a los tres niveles de dificultad baja, media y alta (véase García Madruga, 1984). De ellos, algunos tienen conclusión y proposicional válida y otros no tienen conclusión proposicional válida.

Forma abstracta. Prueba 3A: En el cuadernillo de esta forma se dan unas instrucciones elementales sobre lo que son y cómo se resuelven los silogismos, utilizando algunos ejemplos (que luego no aparecerán en la prueba). En la hoja de respuestas se plantean diez silogismos y se pide al sujeto que dé la conclusión. Los silogismos se presentan utilizando las letras A, B y C. Por ejemplo, se presenta el silogismo "Todo $A$ es $B$ » "Algún $B$ es $C$ » y el sujeto debe escribir la conclusión necesaria que se sigue de las premisas.

Forma con contenido. Prueba 3C: Para esta prueba se crea una situación en la que el sujeto realiza la labor de un antropólogo que está investigando acerca 
de las tribus indias del Norte de América. Se trata de agrupar las distintas tribus en familias según la información fragmentaria de que se dispone. Esta información se presenta en forma de silogismos en los que los términos de cada figura establecen relaciones entre las distintas tribus. El sujeto debe encontrar la conclusión que se sigue necesariamente de cada una de las situaciones. Por ejemplo, se presenta el silogismo «Todos los apaches son sioux» «Algunos sioux son comanches» y el sujeto debe dar la conclusión.

\section{Prueba 4: Tarea de selección}

Esta prueba consiste en la utilización de una regla expresada en términos del condicional. La prueba consta de una sola hoja donde se plantea al sujeto el problema, con todas las instrucciones necesarias, asimismo se le presentan los ítems y debe dar la solución.

Forma abstracta. Prueba 4A: Se utiliza la forma primitiva de esta tarea. Aparecen unas tarjetas con números y letras $(A, 4, B, 7)$, y se propone una regla condicional que establece una relación determinada entre la aparición de los números y las letras (si hay una $\mathrm{A}$ por una cara entonces hay un 4 por la otra cara). El sujeto debe evaluar las tarjetas que se presentan y decidir cuál o cuáles son relevantes para el problema $(¿ Q$ Qué tarjetas hay que levantar para saber si la relación es verdadera?)

Forma con contenido. Prueba 4C: La tarea es formalmente idéntica a la anterior. Aparecen cuatro tarjetas en las que hay personas de distinta edad por una cara y tipos de vehículos por otra. La regla hace referencia a personas mayores o menores de 18 años y a la posibilidad legal de conducir o no un automóvil (si una persona conduce un automóvil entonces debe tener más de 18 años). El sujeto debe escoger las tarjetas relevantes, es decir, aquellas que sería necesario dar la vuelta para comprobar la veracidad de la situación.

\section{TABLA II}

Tipos de pruebas utilizadas, versiones abstractas y concretas

(De acuerdo a esta tabla las pruebas se denominarán $1 \mathrm{~A}, 1 \mathrm{C}, 2 \mathrm{~A}, 2 \mathrm{C}, 3 \mathrm{~A}, 3 \mathrm{C}, 4 \mathrm{~A}$ y $4 \mathrm{C}$, respectivamente.)

\begin{tabular}{lll}
\hline & \multicolumn{1}{c}{ Sin contenido (A) } & Con contenido (C) \\
\hline (1) CONECTIVAS & Montones de fichas con formas y colores & Concurso musical \\
(2) SERIES & Series con letras & Equipos deportivos \\
(3) SILOGISMO & Silogismos con letras & Tribus indias \\
(4) TAREA DE & & \\
SELECCION & Cuatro tarjetas con números y letras & Carnet de conducir \\
\hline
\end{tabular}

En las cuatro pruebas el orden de presentación de los ítems es siempre de menor a mayor dificultad. En la Tabla III, que aparece a continuación, se recoge la descripción de las relaciones lógicas de cada uno de los ítems utilizados en las pruebas 1, 2, 3 y 4 (las formas $\mathrm{A}$ y $\mathrm{C}$ de cada prueba son isomórficas, es decir, tienen la misma estructura lógico-formal). 
TABI.A III

Descripción de las relaciones lógicas de cada uno de los items utilizados en las pruebas 1, 2,3 y 4

1. Conectivas proposicionales

(conjunción): $\mathrm{p}$ y q/items: $\mathrm{p}$ y no $\mathrm{q}$; $\mathrm{p}$ y q; no $\mathrm{p}$ y q; no $\mathrm{p}$ y no q; (disyunción): $\mathrm{p}$ y q/items: $p$ y q; no p y no q; no p y q; p y no q; (doble negación): $p$ y q/items: no $p$ y no q; $p$ y no q; no $p$ y q; $p$ y q; (incompatibilidad): $p$ y q/items: no $p$ y $q ; p$ y $q ; p$ y no q; no p y no q; (bicondicional): $\mathrm{p}$ y q/items: no $\mathrm{p}$ y no q; no $\mathrm{p}$ y $\mathrm{q}$; $\mathrm{p}$ y no q; $\mathrm{p}$ y q; (condicional): $\mathrm{p}$ y q/items: $\mathrm{p}$ y q; $\mathrm{p}$ y no $\mathrm{q}$; no $\mathrm{p}$ y q; no $\mathrm{p}$ y no $\mathrm{q}$.

2. Series de tres términos
1) $\mathrm{B}>\mathrm{C}: \mathrm{B}<\mathrm{A}::<$
2) $\mathrm{C}<\mathrm{B}: \mathrm{B}<\mathrm{A}::<$
3) $\mathrm{B} />\mathrm{A}: \mathrm{C} /<\mathrm{B}:$ : $>$
4) $\mathrm{C}>\mathrm{B}: \mathrm{B} />\mathrm{A}: \mathrm{:}$
5) $\mathrm{B} /<\mathrm{C}: \mathrm{B} />\mathrm{A}::<$
6) $\mathrm{B} />\mathrm{A}: \mathrm{C} />\mathrm{B}::<$
7) $\mathrm{B} /<\mathrm{C}: \mathrm{A} /\langle\mathrm{B}: \mathrm{:}\rangle$
8) $\mathrm{A} /<\mathrm{B}: \mathrm{C} />\mathrm{B}::<$
9) $\mathrm{A} /<\mathrm{B}: \mathrm{B} /<\mathrm{C}:$ : $>$. 10) $\mathrm{A} /<\mathrm{B}: \mathrm{C} />\mathrm{B}::<$.
11) $\mathrm{C}>\mathrm{B}: \mathrm{A} /<\mathrm{B}::<$.
12) $\mathrm{B} /<\mathrm{C}: \mathrm{A} /<\mathrm{B}::<$

3. Silogismos
1) AA-1
2) $\mathrm{EO}-1$
3) $\mathrm{EI}-3$
4) $1 \mathrm{~A}-3$
5) $10-4$
6) $1 \mathrm{~A}-2$
7) $\mathrm{AO}-2$
8) $\mathrm{AI}-2$
9) $\mathrm{AE}-3$
10) EA-3.

4. Tarea de selección

Regla: $p \rightarrow$ q items: $\mathrm{p}, \mathrm{q}$, no $\mathrm{p}$, no q.

\subsection{Procedimiento}

Los sujetos estaban divididos en dos grupos, 1 y 2 . Todos los sujetos realizaron todas las pruebas en el orden comentado anteriormente (véase Tabla I). La administración de las pruebas fue colectiva, realizadas el mismo día y a la misma hora a los dos grupos, a primera hora de la mañana en las aulas naturales. En cada prueba el experimentador leía en voz alta las instrucciones y realizaba los ejemplos mientras los alumnos lo hacían en sus cuadernillos. A continuación se resolvían las posibles dudas y se comenzaba la prueba. No había un tiempo preestablecido para finalizar. El tiempo medio de realización de todo el conjunto de pruebas fue de dos horas, aproximadamente, distribuidas en 5 minutos para cada una de las pruebas $4 a$ y $4 c$, diez minutos para las pruebas 1a, 1c, 2a y $2 \mathrm{c}$, y treinta minutos para las pruebas $3 \mathrm{a}$ y 3 c.

\subsection{Resultados y discusión}

La Tabla IV recoge las puntuaciones medias de los sujetos de los grupos 1 y 2 para cada una de las dos formas de las cuatro pruebas (2). En los gráficos siguientes $(1,2,3$ y 4$)$ aparece una representación de las ejecuciones de los sujetos, expresadas en puntuaciones medias, para cada una de las versiones de las cuatro pruebas. Asimismo se recogen cuatro gráficos, en los que aparece la representación de las puntuaciones para cada una de las cuatro pruebas. 
TABI.A IV

Puntuaciones medias del número de aciertos (Véase la nota 1)

\begin{tabular}{lcc}
\hline & Grupo 1: C-A & Grupo 2: A-C \\
\hline 1 (conectivas) & $3.132-4.395$ & $3.486-2.973$ \\
2 (series) & $11.421-11.632$ & $11.486-11.486$ \\
3 (silogismos) & $3.053-3.211$ & $2.784-3.324$ \\
4 (selección) & $0.895-0.079$ & $0.027-0.297$ \\
\hline
\end{tabular}

Figura 1

Conectivas

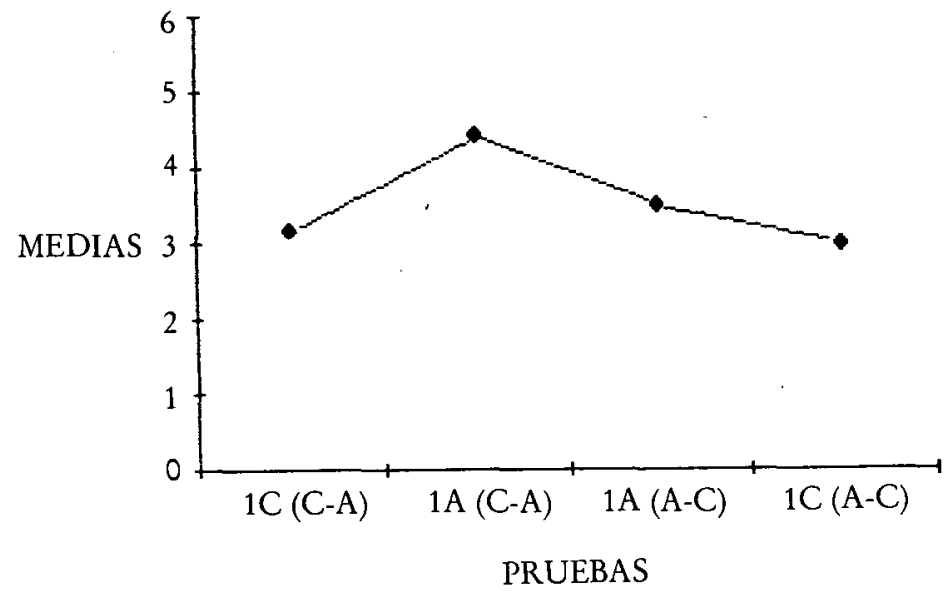

Figura 2

Series

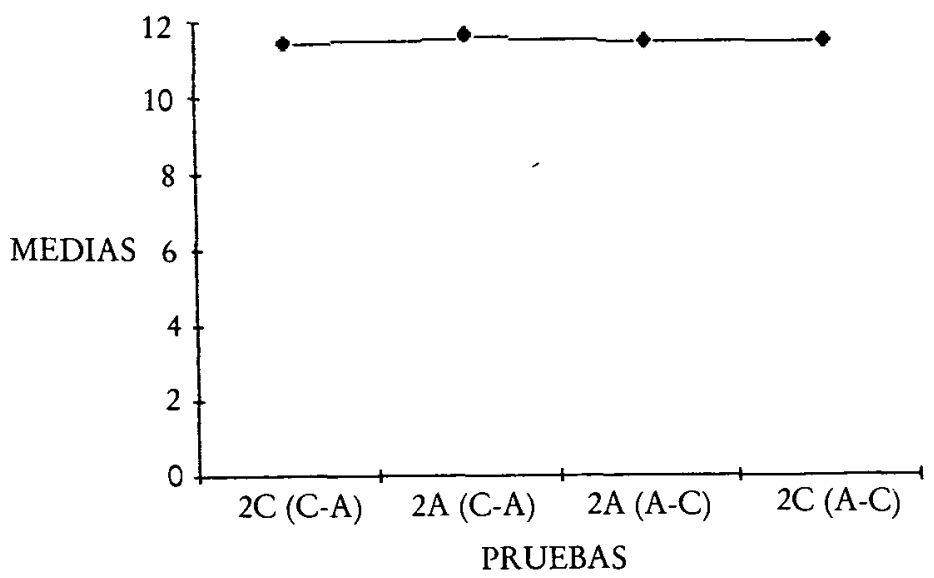


Figura 3

Silogismos

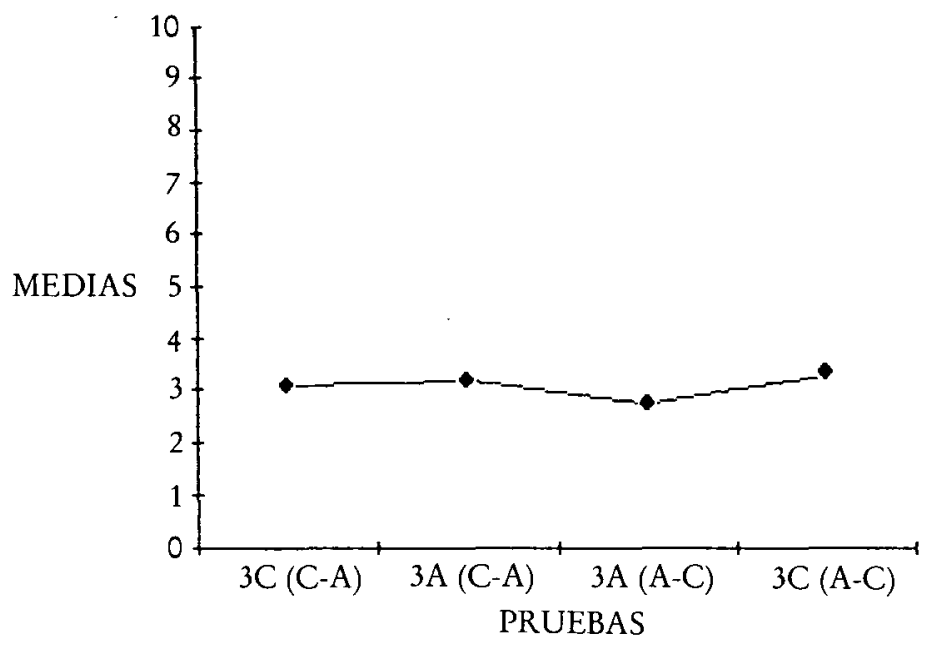

Figura 4

Selección

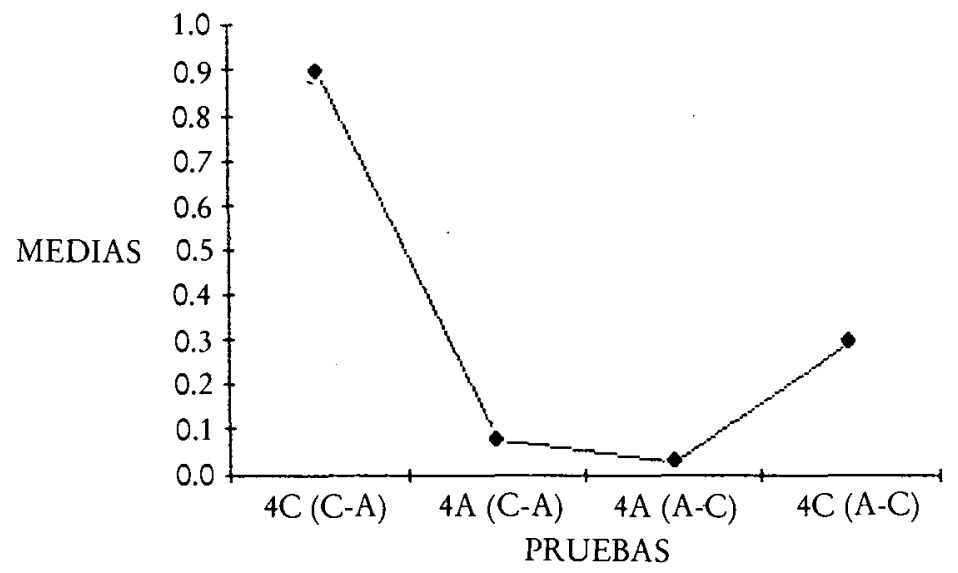

En general, respecto a nuestra primera hipótesis general, podemos confirmar que los sujetos ejecutaron las diversas tareas del mismo modo que se describían en trabajos anteriores. Tanto las dificultades absolutas y relativas de las tareas como las dificultades de los diferentes ítems propuestos se correspondían con los resultados habituales en estas tareas. En la primera prueba (conectivas), que resultó de una dificultad global media, los errores cometidos por los sujetos afectaron fundamentalmente a las conectivas de mayor dificultad (bicondicional y condicional), mientras que todos los sujetos resolvieron 
adecuadamente las conectivas más fáciles (conjunción, disyunción y doble negación). Las diferencias entre los grupos se observaron en cuanto a la solución de la incompatibilidad y en menor medida en cuanto al bicondicional y el condicional. Las ejecuciones de cada uno de los sujetos eran coherentes con el gradiente de dificultad de las conectivas, es decir, fueron prácticamente inexistentes los sujetos que cometían errores en las conectivas de baja dificultad y acertaban las conectivas de alta dificultad. En la segunda prueba (serie de tres términos) puede observarse un efecto techo, la mayor parte de los sujetos resolvieron adecuadamente todos los ítems, incluidos los de alta dificultad. Los escasos errores cometidos en esta prueba no se concentraron en ningún ítem concreto. En la tercera prueba (silogismos) la tarea resultó de gran dificultad para los sujetos. Prácticamente se puede hablar de un efecto suelo en esta tarea si tenemos en cuenta que la mayor parte de los sujetos resolvían adecuadamente tan sólo aquellos ítems de baja dificultad mientras que fallaban sistemáticamente en los ítems de dificultad media y alta. No obstante, todos los sujetos resolvieron adecuadamente aquellos silogismos más fáciles. Por último, en la cuarta tarea (tarea de selección) es donde se estableció una más clara diferencia entre las versiones abstracta y concreta. La versión abstracta resultó de extrema dificultad para los sujetos, mientras que la tarea concreta resultó mucho más fácil.

En la Figura 5, que aparece a continuación, se puede observar una comparación entre las dificultades medias relativas de cada una de las pruebas. En dicha figura solamente se recogen las versiones abstractas y concretas realizadas en primer lugar en cada uno de los grupos, ya que, como luego veremos, las versiones realizadas en segundo lugar se ven afectadas por las primeras. Se observará que las pruebas resultan de una complejidad muy diferente, tal como era esperable en función de trabajos experimentales anteriores. Para sujetos de esta edad y con una configuración de dificultad de los ítems como la seleccionada, la prueba más sencilla resulta la de series, seguida de la prueba de conectivas, posteriormente la prueba de silogismos y, por último, la prueba más difícil resulta la tarea de selección en su versión abstracta. Por el contrario, esta misma prueba en su versión concreta resulta de una dificultad similar a la de series.

Figura 5

Dificultad relativa

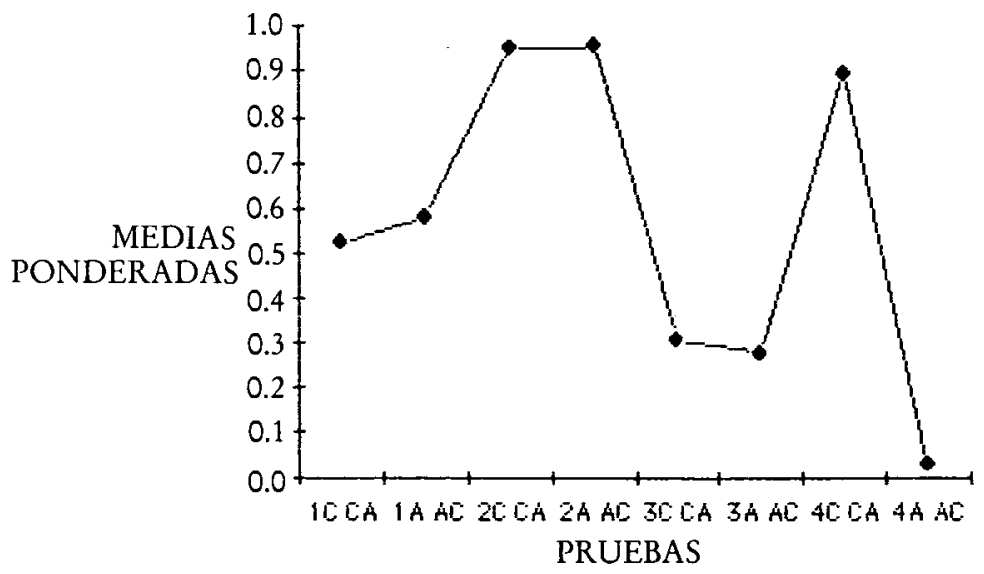


Sobre las puntuaciones medias obtenidas se aplicaron una serie de tratamientos estadísticos para obtener la significación de las diferencias entre las medias de las diferentes pruebas para los dos grupos. Los resultados de los tratamientos aplicados se resumen en la Tabla V.

En primer lugar se estudiaron las diferencias entre la versión concreta y la versión abstracta para cada uno de los tipos de tarea. Se comparó la ejecución de los sujetos del grupo 1 en la primera forma realizada de cada prueba (versión concreta «C») con la de los sujetos del grupo 2 también en la primera forma realizada de cada prueba (versión abstracta «A»).

\section{TABLA V}

Tabla de probabilidades de las diferencias de medias

(Las probabilidades significativas están en negrita)

Comparaciones de la versión abstracta y de la versión concreta, aplicadas ambas en primer lugar a los dos grupos
$1 \mathrm{~A} 1 \mathrm{C}$
$2 \mathrm{~A} 2 \mathrm{C}$
$3 \mathrm{~A} 3 \mathrm{C}$
$4 \mathrm{~A} 4 \mathrm{C}$
0.000
0.408
0.193
0.000

Comparaciones de las versiones abstractas, aplicadas en primero y segundo lugar, respectivamente
$1 \mathrm{~A} 1 \mathrm{~A}$
2A $2 \mathrm{~A}$
$3 \mathrm{~A} 3 \mathrm{~A}$
4A 4A
0.000
0.367
0.125
0.000

Comparaciones de las versiones concretas, aplicadas en primero y segundo lugar, respectivamente
$1 \mathrm{C} 1 \mathrm{C}$
2C $2 \mathrm{C}$
$3 \mathrm{C} 3 \mathrm{C}$
$4 \mathrm{C} 4 \mathrm{C}$
0.000
0.373
0.213
0.000

La versión con contenido resulta claramente más fácil que la versión abstracta para la prueba 4 , existiendo una diferencia muy significativa. Por el contrario, en la prueba 1 existe una diferencia menor pero significativa a favor de la prueba abstracta. En la prueba 3 no hay diferencias significativas entre ambas versiones, aunque se observa una leve tendencia a favor de la versión concreta. En la prueba 2 no existen prácticamente diferencias entre ambas versiones. Parece evidente que en esta última tarea el efecto techo provoca que no existan diferencias entre los grupos. Algo similar ocurre con la tarea de silogismos, ya que, como ya comentamos, hay un efecto suelo para los ítems de dificultad media y alta, por lo cual tampoco aparecen diferencias entre las versiones abstracta y concreta.

Los datos muestran una influencia selectiva del contenido sobre las tareas de razonamiento, lo cual coincide con algunos datos encontrados anteriormente en los que también se describía un efecto facilitador/perjudicador del contenido en las tareas del razonamiento. En la tarea 4 el contenido resulta claramente facilitador. Por el contrario en la tarea 1 dificulta la solución de la prueba. Mientras que resulta irrelevante en las tareas 2 y 3. Estos datos parecen indicar que los sujetos solucionan las pruebas aplicando una serie de habilidades propias para cada tarea y que pueden ser independientes. del contenido de la misma, lo cual explicaría los aciertos y la comisión de errores sistemáticos en ambas formas de la misma tarea de manera independiente del contenido. Sin embargo, no parece que sea ésta la única base de solución de las mismas. Si la 
ejecución mejora o empeora cuando se trabaja sobre un contenido concreto hay que presuponer la existencia de la activación de unos esquemas declarativos que interactúen con la activación de las habilidades lógicas o esquemas de reglas.

Estos resultados apoyarían la existencia de un doble procesamiento. De una parte parecen existir una serie de habilidades de razonamiento lógico que el sujeto es capaz de activar independientemente del contenido de la tarea. El dominio de estas habilidades explicaría tanto los aciertos como los errores sistemáticos que los sujetos cometen en las diferentes tareas de razonamiento. Estas habilidades serían específicas de cada una de las tareas (véase más adelante), y consecuentemente los errores y aciertos son diferentes para cada una de las distintas tareas. Por otro lado el sujeto es capaz también de activar una serie de esquemas de contenido que permiten solucionar la tarea a determinado nivel de complejidad, y que orienta la resolución en determinadas direcciones. Estos esquemas de contenido podrían conducir en determinadas tareas tanto a aciertos como a la comisión de errores sistemáticos. La activación de esquemas de reglas o habilidades procedurales de razonamiento lógico y la activación de esquemas de contenido sería una activación en paralelo. $\mathrm{La}$ solución adecuada de una tarea de razonamiento dependería de la interacción de ambas vías de procesamiento. El acierto o los errores dependerán básicamente de la complementariedad en la dirección de ambas vías.

En segundo lugar se estudiaron las diferencias entre las distintas versiones presentadas en orden inverso. Primero: la influencia de la realización de la versión abstracta después de la realización de la versión concreta, comprando las ejecuciones en las versiones abstractas de los sujetos del grupo 1 con las de los sujetos del grupo 2 (es decir, comparando $1 \mathrm{~A}$ de A-C con $1 \mathrm{~A}$ de C-A). Los resultados aparecen también en la Tabla $\mathrm{V}$. Como puede observarse en la tabla, en las tareas 2 y 3 las ejecuciones de los sujetos en una versión abstracta no se ven ni favorecidas ni perjudicadas significativamente por la ejecución anterior de una tarea con contenido concreto. En las tareas 1 y 4 se observan unas diferencias significativas entre las versiones abstractas. Cuando se realiza delante la versión concreta la tarea abstracta se ve favorecida por la ejecución anterior. Segundo: la realización de la versión concreta después de la realización de la versión abstracta, comparando las ejecuciones en la versión concreta de los sujetos del grupo 2 con las de los sujetos del grupo 1 (es decir, $1 \mathrm{C}$ de C-A con $1 \mathrm{C}$ de A-C). Los resultados aparecen también en la Tabla IV. En la tabla se puede observar que para las tareas 2 y 3 no se producen diferencias significativas. Por el contrario, en las tareas 1 y 4 se produce un efecto negativo para la versión concreta cuando va detrás de la versión abstracta. En suma, teniendo en cuenta las diferencias significativas encontradas, la realización de una versión concreta se ve perjudicada por la realización previa de una versión abstracta y, por el contrario, la realización de una versión abstracta se ve favorecida por la ejecución previa de una versión concreta.

Estos resultados confirman, en primer lugar, nuestro presupuesto básico. La ejecución de una tarea, sea en su versión concreta o abstracta, modifica la ejecución de los sujetos en tareas isomórficas posteriores. Si existieran procesamientos independientes para la solución de ambos tipos de tareas, la ejecución anterior, sea cual fuere, no influiría en la posterior. El hecho de que exista esta influencia indica que parte de la activación producida para la solución de la primera tarea se mantiene activada para la ejecución de la segunda. El problema 
consiste ahora en establecer la direccionalidad de esta influencia respecto al polo abstracto-concreto. Los resultados indican que mientras que una tarea abstracta se ve facilitada por la ejecución anterior de una tarea concreta, la ejecución de una tarea concreta se ve dificultada por la ejecución anterior de una tarea abstracta (ambos efectos observados sobre las tareas 1 y 4). Este resultado iría en la línea de presuponer una primacía del procesamiento semántico-pragmático sobre el procesamiento sintáctico. Así, los sujetos que son capaces de activar un modelo o esquema para solucionar determinada tarea parecen ser capaces de utilizar el mismo para la solución de una tarea en la que dicho esquema o modelo no viene explícitamente activado. Por el contrario, los sujetos que son capaces de activar una regla para solucionar un problema abstracto no parecen verse beneficiados en la solución posterior de un problema concreto, más bien la activación de esta regla general parece confundir la ejecución posterior de un problema donde el contenido juega un papel relevante.

En tercer lugar se estudió la relación entre las ejecuciones de los sujetos en las distintas tareas de razonamiento presentadas. De una parte se hallaron las correlaciones entre las versiones abstractas y concretas en ambos grupos de sujetos. Y de otra, se hallaron las correlaciones entre las diferentes pruebas de razonamiento, de un lado las existentes entre las pruebas concretas en el grupo primero y de otro la relación entre las pruebas abstractas en el grupo segundo. Los resultados se recogen en la Tabla VI, que aparece a continuación.

\section{TABLA VI}

Posibilidades encontradas para las correlaciones entre las diferentes pruebas (Las probabilidades significativas están en negrita)

Significaciones de las correlaciones entre las versiones abstracta y concreta, para cada una de las pruebas, en la situación $\mathrm{A}-\mathrm{C}$
$1 \mathrm{~A} 1 \mathrm{C}$
$2 \mathrm{~A} 2 \mathrm{C}$
$3 \mathrm{~A} 3 \mathrm{C}$
4A 4C
0.587
0.020
0.005
0.057

Significaciones de las correlaciones entre las versiones abstracta y concreta, para cada una de las pruebas, en la situación C-A
$1 \mathrm{C} 1 \mathrm{~A}$
$2 \mathrm{C} 2 \mathrm{~A}$
$3 \mathrm{C} 3 \mathrm{~A}$
$4 \mathrm{C} 4 \mathrm{~A}$
0.070
0.000
0.005
0.267

Significaciones de las correlaciones:

entre las versiones abstractas

\begin{tabular}{|c|c|c|c|c|c|c|c|}
\hline & & & & & & & \\
\hline & $2 \mathrm{~A}$ & $3 \mathrm{~A}$ & $4 \mathrm{~A}$ & & $2 \mathrm{C}$ & $3 C$ & $4 C$ \\
\hline $1 \mathrm{~A}$ & 0.000 & 0.781 & 0.189 & $1 \mathrm{C}$ & 0.025 & 0.943 & 0.206 \\
\hline $2 \mathrm{~A}$ & & 0.219 & 0.363 & $2 \mathrm{C}$ & & 0.071 & 0.687 \\
\hline $3 A$ & & & 0.617 & $3 \mathrm{C}$ & & & 0.158 \\
\hline
\end{tabular}

En el caso de las comparaciones de las versiones abstractas y concretas, tanto para el grupo 1 como para el grupo 2, los resultados indican que no existe correlaciones significativas para las pruebas 1 y 4 . Las correlaciones resultan significativas para las pruebas 2 y 3 . Estos resultados son consistentes con los comentados anteriormente. Recordemos que en las pruebas 2 y 3 los sujetos resolvían la tarea de una manera prácticamente igual (en serie porque todos lo hacían bien y en silogismos porque todos hacían bien los de baja 
dificultad y mal los de media y alta). Por tanto era esperable encontrar correlaciones significativas debido a estos efectos. Sin embargo resultan más relevantes los resultados de las pruebas 1 y 4 , debido a que en ellas sí existió una variabilidad mayor en las respuestas de los sujetos. Los resultados indican que no existe correlación entre ambas versiones, concreta y abstracta, de la misma prueba. Si los sujetos activaran para ambas versiones el mismo tipo de habilidad de razonamiento para ambas versiones, las correlaciones deberían ser positivas y significativas. El hecho de que no ocurra esto parece indicar que, por el contrario, los sujetos activan diferentes estrategias de solución del problema cuando se presentan ante las diferentes versiones de la misma prueba. Estos resultados irían en contra de la presunción de la existencia de una habilidad de razonamiento general que nosotros activamos cuando nos enfrentamos a un determinado tipo de tarea. No parece que seamos capaces de activar estas habilidades generales ni aun en este caso en que ambas pruebas presenten una estructura lógico-formal isomórfica.

En el caso de las comparaciones de las diferentes pruebas de razonamiento planteadas tanto para las versiones abstractas como para las concretas, los resultados indican que no existen correlaciones significativas entre las diferentes pruebas, a excepción de la prueba 1 con la prueba 2 . Si nosotros presuponemos la existencia de una estructura general de competencia del razonamiento humano deberían existir correlaciones significativas y positivas entre las diferentes pruebas de razonamiento. Los datos demuestran que esto no es así no sólo para las pruebas concretas, sino también para las pruebas abstractas. Aun en el caso de que las pruebas de razonamiento se basen en un análisis lógico-formal muy similar, como ocurre en la prueba 4 con algunos ítems de la prueba 1 , o entre las pruebas de silogismos lineales y categoriales ( 2 y 3 ). Por tanto no parece sostenible la hipótesis de la existencia de estas estructuras generales que orienten el razonamiento con independencia del tipo de tarea y del tipo de contenido de la misma. Al contrario, parece cobrar fuerza la hipótesis anteriormente comentada, es decir, la existencia de un repertorio de habilidades de razonamiento que el sujeto va dominando progresivamente y que es capaz de activar selectivamente en función de la demanda concreta de cada una de las tareas planteadas.

\section{CONCLUSIONES E IMPLICACIONES}

Reordenemos las conclusiones que pueden apuntarse a partir de los resultados obtenidos. Antes que nada, es preciso señalar que los resultados que aquí se recogen son parciales, sobre todo si se tiene en cuenta la importancia de las cuestiones que se discuten. Por tanto, las reflexiones que vamos a realizar tienen que ver con algunas tendencias que parecen apuntarse a partir de nuestros resultados y de trabajos anteriores. Es indudable la necesidad de seguir profundizando en el estudio cada vez más detallado de estas cuestiones.

La cuestión crucial radica en que habitualmente se ha supuesto que la teoría de la competencia en el razonamiento lógico debía adoptar la forma de alguna lógica mental. Las teorías actuales sobre el razonamiento, como los modelos mentales, son teorías de la competencia que no presuponen la existencia de una lógica mental. Otra cuestión es que existan niveles de computación que no estén basados en ningún aspecto declarativo por lo que 
los sujetos no puedan activar el componente semántico. En estos casos los sujetos pueden resolver los problemas aplicando unas habilidades procedurales propias de un componente sintáctico. Pero estas situaciones se corresponden con un nivel algorítmico o de rendimiento de la teoría. Dicho de otro modo, si desechamos que la teoría de la competencia del razonamiento deductivo haya de tomar la forma de alguna lógica mental, lo que nos queda es definir si el procesamiento es, en este plano teórico de la competencia, de tipo exclusivamente semántico (tal como propone, por ejemplo, la teoría de los modelos mentales), o si además habría que incluir niveles de procesamiento superficial en los que la solución de la tarea estaría guiada por una «representación pobre», tal como ocurre en algunos casos de razonamiento logístico (García Madruga y Carretero, 1988). Una alternativa probable puede consistir en concebir la solución de tareas de razonamiento como un procesamiento en paralelo de los diferentes componentes, los cuales determinan su resultado en la medida en que alcanzan los niveles de activación necesarios. De este modo, si el componente semántico no ha resultado suficientemente activado puede activarse un componente sintáctico, representación pobre que conducirá necesariamente a una solución basada en un procesamiento superficial.

El hecho de que los sujetos resuelvan de modo diferenciado dos versiones isomórficas de la misma tarea con independencia de su cualidad virtual, estaría muy lejos de indicar que se puede presuponer que la ejecución de los sujetos se basa exclusivamente en la existencia de una estructura general de carácter exclusivamente sintáctico. Por el contrario, habría que presuponer que el sujeto es capaz de activar primordialmente una serie de modelos o esquemas de contenido que tiene en cuenta para solucionar el problema. Sin embargo, los sujetos resuelven en ocasiones tareas carentes de contenido. Sin embargo, estos resultados encontrados podrían basarse en la existencia de un procesamiento en paralelo, tanto de tipo sintáctico, que permitiría explicar las consistencias existentes en las ejecuciones de los sujetos respecto a la forma del razonamiento en un amplio conjunto de tareas, como de tipo semánticopragmático, que permitiría explicar las dependencias de las ejecuciones de los sujetos de las características virtuales del contenido de las tareas propuestas.

Una propuesta de este tipo, que implica la interdependencia procesual, permite presuponer la existencia de una estructura general de razonamiento que contempla diversos tipos de procesamiento. Sin embargo, a pesar de que un sistema de razonamiento de estas características permite enmarcar adecuadamente los resultados de diversas líneas de investigación en el razonamiento, no está exento de problemas. En primer lugar, quedarían por especificar las direccionalidades de los diversos tipos de procesamiento. Es decir, qué tipo de características de procesamiento se aplican en uno u otro caso. En segundo lugar quedaría por especificar la primacía de los diversos procesamientos. Es decir, si los procesamientos desarrollados por el sujeto son convergentes, hacia la facilidad o hacia la dificultad, no se plantearían problemas más allá de la solución adecuada o inadecuada de la tarea; pero qué ocurre si son divergentes, si ante una determinada tarea el procesamiento sintáctico provoca una direccionalidad opuesto al procesamiento semántico-pragmático. Disponemos en esta misma investigación, y en otras, de algunos datos en este sentido: parece existir una primacía del procesamiento semántico-pragmático respecto al procesamiento sintáctico.

A la vista de la discusión que estamos argumentando parece claro que 
estamos aún lejos de poder ofrecer unas implicaciones unívocas que vayan más allá de una serie de reflexiones generales. Sin embargo, desde hace mucho tiempo, a raíz de determinadas posiciones teóricas e investigaciones desarrolladas en el campo del razonamiento, se han apuntado una serie de aplicaciones que han orientado la intervención, fundamentalmente en el campo educativo. La mayoría de estas intervenciones se han basado en la presuposición de la existencia de un procesamiento sintáctico, la mayor parte de tipo general y en algunos casos de tipo específico (véase, por ejemplo, la revisión de Nickerson, Perkins y Smith, 1985; para una revisión de esquemas pragmáticos véanse los trabajos de Cheng, Holyoak, Nisbett y Oliver, 1986). En consecuencia, estos programas no recogerían los aspectos relativos al procesamiento semánticopragmático, lo cual, evidentemente, implica no recoger la experiencia previa de los sujetos en los diferentes dominios específicos de conocimiento. La idea básica de estos programas es muy simple: una vez presupuestas las reglas del sistema general de razonamiento se trata de implementar el aprendizaje de dichas reglas. Estos programas de intervención se están aplicando en muy diversos contextos con una escasa reflexión sobre los presupuestos de base y sobre las condiciones de aplicabilidad en cada caso.

No parece que los más recientes datos de investigación, entre ellos los que acabamos de exponer, avalen los presupuestos de base de estos programas. Dos conclusiones de la discusión que hemos sostẹido son relevantes para esta valoración. De una parte, dejar de lado el aspecto de componente semántico-pragmático desvirtuaría la mayor parte de los razonamientos donde podemos presuponer que existe una presencia importante de este componente, a saber: la vida cotidiana y aquellas tareas escolares o profesionales con un contenido temático específico donde el sujeto tiene una experiencia sensible que le permite generar unos modelos o esquemas que orienten sus inferencias. De otra parte, no parece que se pueda presuponer una primacía del procesamiento sintáctico sobre el procesamiento semántico-pragmático; más al contrario, la activación de reglas generales no parece beneficiar la activación posterior de modelos o esquemas en tareas particulares. En suma, la «conclusión» de que los programas instruccionales que presuponen habilidades sintácticas generales, tan de moda actualmente, no podemos decir que se siga necesariamente de las actuales «premisas» de la psicología del razonamiento. 


\section{Referencias}

Beth, E. W. y Piaget, J. (1961) Epistémologie mathématique et Psychologie, París: PUF. Trad. cast. de V. Sánchez: Relaciones entre la lógica formal y el pensamiento real. Madrid: Ciencia Nueva, 1968.

BraclewELL, R. J. y Hidi, S. E. (1974) The solution of an inferential problem as a function of the stimulus materials. Quaterly Journal of Experimental Psychology, 26, 480-488.

BrAint. M. D. S. (1978) On the relation between the natural logic of reasoning and standard logic. Psychological Review, 85, 1-21.

Brain, M. D. S. y Rumain, B. (1983) Logicl Reasoning. En: P. H. Mussen (Ed.) Child Development. Vol. III: Cognitive Development. N.Y.: Wiley y Sons, pp. 263-340.

Byrnes. J. P. y Overton. W. F. (1986) Reasoning about certainty and uncertainty in concrete, causal, and propositional contexts. Development Psychology, 22, 793-799.

Byrnes. J. P. y Overton, W. F. (1988) Reasoning about logical connectives: A developmental analysis. Journal of Experimental Child Psychology, 46, 194-218.

Cheng, P. W. y Hollyoak, K. J. (1985) Pragmatic reasoning schemas. Cognitive Psychology, 17, 391-416.

Cheng, P. W., Hollyonk, K. J., Nisbett, R. E. y Oliver. L. M. (1986) Pragmatic versus syntactic approaches to training deductive reasoning. Cognitive Psychology, 18, 293-328.

CoHen, L. J. (1981) Can human irrationality be experimentally demonstrated?, The Behavior and Brain Sciences, 4, 317-331.

Donaldson, M. (1976) Development of Conceptualization. En: V. Hamilton y M. D. Veron (Eds.) The Development of Cognitive Processes. N.Y.: Academic Press.

Fong, G. T., Krantz, D. H. y Nisbett, R. E. (1986) The effects of statistical training on thinking about everyday problems. Cognitive Psychology, 18, 253-292.

García Madruga. J. A. (1984) Procesos de error en el razonamiento silogístico: doble procesamiento y estrategia de verificación. En: M. Carretero y J. A. García Madruga (Eds.) Lecturas de Psicología del Pensamiento. Madrid: Alianza, pp. 113-121.

García Madruga, J. A. y Carretero, M. (1986) Estrategias en el Pensamiento Humano: tareas lógicas y probabilísticas. En H. Peraita (Ed.) Psicología Cognitiva y Ciencia Cognitiva, Madrid: UNED, pp. 171-204.

Grice, H. P. (1975) Logic and Conservation. En: P. Cole y J. L. Morgan (Eds.). Syntax and Semantics. Vol. 3: Speech Acts. N.Y.: Academic Press.

Grice, H. P. (1978) Further notes on logic and conservation. En: P. Cole (Ed.). Syntax and Semantics. Vol. 9: Pragmatics. N.Y.: Academic Press.

Griggs, R. A. y Cox, J. R. (1982). The elusive thematic-materials effects in Wason's selection task, British Journal of Psychology, 73, 407-420.

Griggs, R. A. (1983) The role of problem content in the selection task and the thog problem. En: J. St. B. T. Evans (Ed.) Thinking and Reasoning. Psychological Approches. Londres: Routledge y Kegan Paul, pp. 16-43.

Henle, M. (1962) On the relation between logic and thinking, Psychological Review, 69, 366-378. Trad. cast. de J. Delval en J. Delval (Ed.) Investigaciones sobre lógica y psicología. Madrid: Alianza, 1977, pp. 43-60.

Henle, M. (1978) Prólogo de Revlin, R y Mayer, R. E. (Eds.) Human Reasoning, Washington, D.C.: Winston.

HENLE, M. y MiCHAEL, M. (1956) The influence of attitudes on syllogistic reasoning. The Journal of Social psychology, 44, 115-127. Trad. cast. de J. Delval en J. Delval (Ed.) Investigaciones sobre lógica y psicología. Madrid: Alianza, 1977, pp. 176-190.

Holland, J. H., Hollyoak. K. J., Nisbett, R. E. y Thagard, P. R. (1987) Induction: Processes of Inference, Learning and Discovery. Londres: The MIT Press.

Johnson-Laird, P. N. (1983) Mental Models. Cambridge, MA: Harvard University Press.

Johnson-Laird, P. N. (1988) The Computer and the Mind: An Introduction to Cognitive Science. Londres: Fontana.

Johnson-Laird, P. N., Legrenzi, P. y Sonino Legreñz, M. (1972) Reasoning and a sense of reality. British Journal of psychology, 63, 395-400.

LEFFORD, A. (1946) The influence of emotional subject matter on logical reasoning. Journal of General Psychology, 30, 127-151.

Manktelow, K. I. y Evans, J. St. B. T. (1979) Facilitation of reasoning by realism: Effect or non effect?. British Journal of Psychology, 70, 477-488.

Morgan, J. J. y Morton, J. T. (1944) The distorsion of syllogistic reasoning produced by personal convictions. The Journal of Social Psychology, 20, 39-59. Trad. cast. de J. Delval en J. Delval (Ed.) Investigaciones sobre lógica y psicología. Madrid: Alianza, 1977, pp. 148-175.

Nickerson, R. S., Perkins, D. N. y Smith, E. E. (1985) The teaching of the thinking. N.J.: LEA. Trad. cast. de L. Romano y C. Ginard: Enseñar a pensar: aspectos de la aptitud intelectual. Barcelona: Paidos, 1987. 
O'BRII:N, D y OVERTTON, W. F. (1982) Conditional reasoning following contradictor y evidence: A developmental analysis. Journal of Experimental Child Psychology, 30, 44-61.

O'BRII:N, D. y OVI:R'TON, W. F. (1982) Conditional reasoning and the competence-performance issue: A developmental analysis of a training trask. Journal of Experimental Child Psychology, $34,274-290$.

Oshi:RSON. D. N. (1975) Logic and models of logical thinking. En: R. J. Falmagne (Ed.) Reasoning: representation and process in children and adults. Hillsdale, N.J.: I.EA.

OvirTon. W. F., Ward. S. L., Novick, I. A., Bt.Ack, J. y O'Brilin. D. P. (1987) Form and Content in the Development of Deductive Reasoning. Developmental Psychology, 23, 1, 2230.

PIAGFT. J. (1970) L'evolution intellectuelle entre l'adolescence et l'age adulte. Rapport sur le III Congrés International FONEME. Milan, pp. 149-156. Trad. cast. de J. Delval en J. Delval (Ed.) Lecturas de Psicología del niño. Madrid: Alianza, 1978, pp. 208-213.

POI.l.ARD, P. (1981) The effect of thematic content in the Wason selection task. Current Psychological Research, 1, 21-29.

RII'S, L. J. (1983) Cognitive processes in propositional reasoning. Psychological Review, 90, 38-71. RII's. J. L. y Marcus, S. L. (1977) Suppositions and the analysis of conditional sentences. En: M. A. Just y P. A. Carpenter: Cognitive Processes in Comprebension. Hillsdale, N.J.: LEA.

VAN DUYN1, P. C. (1974) Realism and linguistic complexity in reasoning. British Journal of Psychology, 65, 59-67.

WASON. P. C. y SHAPIRO. D. (1971) Natural and contrived experience in a reasoning problem, Quaterly of Experimental Psychology, 23, 63-71.

\section{Notas}

(1) Se presentan las medias en vez de las medianas, a pesar del carácter poco rígido de las condiciones de medición debido a que el excesivo número de ligas desdibujaba las diferencias entre los tratamientos en el caso de utilizar las medianas; no obstante, todos los tratamientos estadísticos posteriores son no paramétricos. En función del carácter de las puntuaciones se utilizaron diferentes estadísticos para las distintas pruebas. Para hallar las diferencias de medias se utilizaron al U de Mann-Witney y la diferencia de proporciones. Para hallar las correlaciones se utilizaron la correlación de Spearman y el coeficiente Phi. 